\title{
$\angle S$ Research Square \\ Genome-wide Identification and Characterization of Perirenal Adipose Tissue MiRNAs in Rabbits Fed With a High-fat Diet
}

\section{Jie Wang}

Sichuan Agricultural University

Jiahao Shao

Sichuan Agricultural University

Yanhong Li

Sichuan Agricultural University

Mauricio A. Elzo

University of Florida

Xianbo Jia

Sichuan Agricultural University

Songjia Lai ( $\sim$ laisj5794@163.com )

Farm Animal Genetic Resources Exploration and Innovation Key Laboratory of Sichuan Province, Sichuan Agricultural University, Chengdu, Sichuan 611130, China

\section{Research}

Keywords: Adipogenesis, High-fat diet, MiRNAs, Rabbits

Posted Date: July 28th, 2020

DOl: https://doi.org/10.21203/rs.3.rs-44842/v1

License: (c) (1) This work is licensed under a Creative Commons Attribution 4.0 International License. Read Full License 


\section{Abstract \\ Background}

MicroRNAs (miRNAs) are a class of endogenous single-stranded RNA molecules that play an important role in gene regulation in animals by pairing with target gene mRNAs. However, the functions of miRNAs in the adipogenesis of obese rabbits are poorly understood.

\section{Methods}

Six small RNA libraries from rabbits under a standard normal diet (SND; $n=3$ ) and a high-fat diet (HFD; $n$ $=3$ ) were constructed and sequenced. Differentially expressed (DE) miRNAs were identified using the edgeR data analysis package from R. Software miRanda and RNAhybrid were used to predict the target genes of miRNAs. To further explore the functions of DE miRNAs, Gene Ontology (GO) and Kyoto Encyclopedia of Genes and Genomes (KEGG) pathway enrichment analysis were performed.

\section{Results}

A total of 69577441 clean reads were obtained from the six libraries, of which, 52 known DE miRNAs (24 up-regulated, 28 down-regulated) and 31 novel DE miRNAs (14 up-regulated, 17 down-regulated) were identified. GO enrichment analysis revealed that the DE miRNAs target genes were involved in intermediate filament cytoskeleton organization, intermediate filament-based process, and alpha-tubulin binding. DE miRNAs were involved in p53 signaling, linoleic acid metabolism, and other adipogenesisrelated KEGG pathways.

\section{Conclusions}

Our study further elucidates the possible functions of DE miRNAs in rabbit adipogenesis, contributing to the understanding of rabbit obesity.

\section{Background}

Obesity is spreading rapidly in both developed and developing countries, becoming a considerable public health concern threatening human health [1]. According to the definition of obesity (body mass index (BMI) higher than $30 \mathrm{~kg} / \mathrm{m}^{2}$ ) by the World Health Organization (WHO), females suffer a higher incidence of obesity than males, and this phenomenon tends to occur at a younger age [2,3]. The global epidemic of obesity stems from the increased adipose mass that results from an imbalance between dietary energy intake and consumption [4]. White adipose tissue (WAT) is widely distributed in subcutaneous tissues and around internal organs, which play a wide range of functions in metabolic regulation and 
physiological homeostasis, far beyond the simple model of fat storage [5]. Excessive accumulation of WAT is considered to be a risk factor for morbid obesity and some obesity-related diseases [6, 7].

The underlying mechanism of obesity is a multifactorial pathological process that involves the interaction between genes and the environment [8]. MiRNAs regulation has been confirmed to play an important role in the development of obesity. For example, miR-125a-5p promotes proliferation and inhibits differentiation of adipocytes by kruppel like factor 13 (KLF13) [9]. MiR-148a-3p significantly upregulates the mRNA levels of PPARY, C/EBPa, and FABP4, and promotes intracellular triglyceride accumulation [10]. Diet-induced fat in rabbits has a similar lipid metabolism to humans, making rabbits a reliable and low-cost obesity research model animal [11]. However, the molecular mechanisms of adipogenesis by mediating miRNA transcriptomes in high-fat diet-induced obese rabbits requires further research.

The objective of this study was to investigate the role of miRNAs in adipogenesis by sequencing and analyzing perirenal adipose tissue miRNAs from rabbits fed a standard normal diet (SND) and a high-fat diet (HFD) to obtain new insights into miRNAs regulatory functions and contribute to the understanding of epigenetic mechanisms influencing fat metabolism in obese rabbits.

\section{Materials And Methods}

\section{Animals}

The animals in this study were 24 female Tianfu black rabbits from a strain breed at the Sichuan Agricultural University in China that were 35 days of age. Rabbits were randomly divided into two groups and fed either a standard normal diet (SND) or a high-fat diet (HFD; a 10\% lard was added to the standard normal diet) for four weeks. Animals had free access to water and were fed twice a day. Each rabbit was housed in a clean iron cage $(600 \times 600 \times 500 \mathrm{~mm})$ and kept in an environmentally controlled room. At the end of the trial, rabbits were screened for obesity using serum triglycerides (TG) concentration and body fat rate, and three rabbits from each group meeting the obesity requirements were randomly selected for sampling.

\section{Blood Collection and Measurement}

Blood samples from animals fasted overnight were collected the next morning from the auricular vein using vacutainer tubes. Then, blood samples were centrifuged at $4^{\circ} \mathrm{C}$ for 5 minutes and the serum was transferred to clean frozen pipes and stored at $-80^{\circ} \mathrm{C}$. Serum triglyceride concentrations were obtained by personnel of the Lilai Biological Company (Lilai, Chengdu, China).

\section{Adipose tissue sample collection}


Perirenal adipose tissue samples were taken immediately after rabbits were euthanized (Shock and bleed treatment). Tissue blocks were placed in $4 \mathrm{ml} \mathrm{EP}$ tubes and stored in a $-80^{\circ} \mathrm{C}$ freezer. All experimental protocols were performed under the direction of the Institutional Animal Care and Use Committee from the College of Animal Science and Technology, Sichuan Agricultural University, China (DKYB2019202015).

\section{RNA extraction}

Total RNA from samples was extracted using RNAiso Plus Reagent (Invitrogen, Hong Kong, China), following the guidelines of the manufacturer. Subsequently, the purity, concentration, and integrity of RNA were determined by Nanodrop (Thermo Fisher Scientific, Waltham, MA, USA), Qubit 2.0 fluorometer (Life Technologies, Carlsbad, CA, USA) and Agilent 2100 Bioanalyzer system (Agilent Technologies, Carlsbad, CA, USA), and only qualified RNA was used for the trial.

\section{Small RNA library construction and sequencing}

To identify changes in miRNAs from perirenal adipose tissue induced by HFD, we constructed six small RNA libraries (SND-1, SND-2, SND-3, HFD-1, HFD-2, HFD-3) from SND and HFD rabbits. The small RNA libraries were constructed using TruSeq Small RNA Sample Prep Kits (Illumina, San Diego, USA), following the manufacturer's instructions. Small RNA of sizes ranging from 15 to $30 \mathrm{nt}$ were isolated using a 15\% PAGE gel. The 5 ' and 3 ' adaptors were ligated sequentially to the small RNA using a T4 RNA ligase (Promega, USA) and then amplified by q-PCR. Lastly, the libraries were sequenced using the Illumina HiSeq platform, and then 50bp paired-end reads were generated.

\section{Identification of miRNAs}

SOAPnuke software was used to filter the sequencing reads, and after removing the 3 ' containing sequencing adaptors, reads larger than $18 \mathrm{nt}$ (clean reads) were retained. The miRBase was used as a reference database, and software miRDeep2 was used to predict novel miRNAs. The mapped sequences contained some known types of RNA, such as RFam data (tRNA, rRNA, snRNA, snoRNA), repeated sequences, and mRNA degraded fragments. Small RNA reads of $18 \mathrm{nt}$ or greater were aligned to rabbit reference genomes with Bowtie2, and comparisons between multiple databases (Rfam database, genebank database, and repeat database) were used to remove known types of RNA sequences and repeated sequences. Next, the filtered small RNA reads were compared to the miRbase database to identify known miRNAs that could be directly used for subsequent analyses. Software package miRDeep2 was used to identify novel miRNAs from unannotated reads. The edgeR data analysis package from $\mathrm{R}$ was used for differential expression analysis. The threshold was defined to be llog2(fold change) $\mid \geq 1$ and FDR $<0.05$. The known DE miRNAs in the six libraries were clustered using the $R$ function Heatmap.

\section{Target gene prediction and functional enrichment analysis}


Software miRanda and RNAhybrid were used to predict the target genes of miRNAs, and the intersection of the predicted results was taken as the final outcome. Gene Ontology (GO) terms and KEGG (Kyoto Encyclopedia of Genes and Genomes) pathway enrichment analysis of DE miRNAs target genes was performed using online software DAVID Bioinformatics Resources 6.7 (http://david.abcc.ncifcrf.gov/home.jsp).

\section{Validation of DE miRNAs}

Reverse transcription of miRNAs was performed using the Mir- $\mathrm{X}^{\mathrm{TM}}$ miRNA First-Strand Synthesis Kit (TakaRa, Dalian, China), following the manufacturer's protocol. Subsequently, q-PCR was performed in triplicate using the TB Green ${ }^{\mathrm{TM}}$ Premix Ex Taq ${ }^{\mathrm{TM}}$ ॥I (TakaRa, Dalian, China) on a CFX96 instrument (BioRad, USA), and relative expression levels of miRNAs were calculated by $2^{-\triangle \Delta C T}$ method.

\section{Statistical analysis}

SPSS Statistics 20.0 (SPSS Inc., Chicago, IL, USA) was used for statistical analysis. Groups were compared using Student's t-test. Differences were considered to be statistically significant at $P<0.05$.

\section{Results}

\section{Establishment of the rabbit obesity model}

After 4 weeks of trial, the HFD rabbits had higher body weight (Fig. 1a). In addition, perirenal adipose tissue weight was heavier in HFD rabbits (Fig. 1b) than in SND rabbits (Fig. 1c). The serum TG concentration and the body fat rate of SND and HFD rabbits are shown in Fig. 1d and Fig. 1e, respectively. HFD rabbits achieved experimental obesity and could be used for further experiments.

\section{Quality assessment of small RNA sequencing}

After raw reads were quality filtered, a total of 69577441 clean reads were obtained from the six small RNA sequencing libraries. The Q20 (percentage of reads with a Phred quality value $>20$ ) ranged from 99.54 to $99.86 \%$, and the GC content of libraries ranged from 45.74 to $47.72 \%$. The clean reads were mapped to the rabbit reference genome, and the mapped rate ranged from 84.44 to $90.69 \%$ (Additional file 1). Further, the average length of most reads was $22 \mathrm{nt}$ in the six small RNA libraries, which was consistent with the length characteristics of animal miRNAs (Fig. 2a).

\section{Screening for known and novel miRNAs}

Mapped sequences were compared to the Rfam, genebank, and repeat databases for classification and annotation. The number of high-quality miRNA sequences obtained for each sample were 7383340 for SND-1, 6678939 for SND-2, 10137372 for SND-3, 6492570 for HFD-1, 6984965 for HFD-2, and 7292413 for HFD-3 (Table 1). Next, the filtered small RNA reads were perfectly compared to mature rabbit miRNA in 
the miRbase database to identify 671 known miRNAs (Additional file 2). Lastly, 488 novel miRNAs were identified using software package miRDeep2 (Additional file 3).

Table 1 Distribution of total sRNA identified in SND and HFD rabbits ${ }^{1}$

\begin{tabular}{|c|c|c|c|c|c|c|}
\hline & \multicolumn{3}{|l|}{ SND } & \multicolumn{3}{|l|}{ HFD } \\
\hline & SND-1 & SND-2 & SND-3 & HFD-1 & HFD-2 & HFD-2 \\
\hline Mapped sequences & 8900721 & 8481469 & 15537856 & 8730388 & 8904422 & 10084871 \\
\hline miRNA & 7383340 & 6678939 & 10137372 & 6492570 & 6984965 & 7292413 \\
\hline Precursor & 1036389 & 937443 & 554794 & 836396 & 644445 & 1017783 \\
\hline rRNA & 104255 & 79579 & 100251 & 75058 & 79824 & 122625 \\
\hline stRNA & 81618 & 214085 & 131953 & 126184 & 118746 & 117323 \\
\hline snRNA & 501 & 551 & 1073 & 481 & 570 & 532 \\
\hline snoRNA & 72246 & 31235 & 54692 & 23323 & 28657 & 34689 \\
\hline tRNA & 7796 & 3150 & 1422 & 6626 & 2771 & 3269 \\
\hline Unannot & 211560 & 146655 & 470768 & 200255 & 193311 & 289598 \\
\hline Unmap & 332442 & 496160 & 839678 & 415599 & 477062 & 528274 \\
\hline
\end{tabular}

${ }^{1}$ SND = Standard normal diet; HFD = High-fat diet.

\section{Screening of DE miRNAs}

To explore the expression patterns of all known and novel miRNAs in SND and HFD rabbits perirenal adipose tissue, differential expression analyses of the miRNAs were performed using EdgeR. A total of 52 known DE miRNAs (24 up-regulated, 28 down-regulated) and 31 novel DE miRNAs (14 up-regulated, 17 down-regulated) were identified in SND and HFD rabbits (Fig. 3a). Values of log2(fold change) and $\log 10$ (padj) were used to construct volcano figures for known (Fig. 3b) and novel (Fig. 3c) DE miRNAs. Known DE miRNAs with similar expression levels across different libraries were screened and grouped using a hierarchical cluster analysis (Fig. 3d).

\section{Enrichment analysis of known DE miRNAs target genes}

To better study the biological functions of the DE known miRNAs, we used software to obtain 190 known DE miRNAs target genes. GO analysis of known DE miRNAs target genes found a total of 1232 enriched GO terms (885 biological processes (BP), 164 cellular components (CC), and 183 molecular functions (MF)) (Additional file 4). Multiple significance tests showed that 371 out of $1232 \mathrm{GO}$ terms $(30.11 \%)$ were significantly enriched $(P<0.05)$. The main biological processes involved in known DE miRNAs target genes included focal adhesion, alpha-tubulin binding, and regulation of cytoskeleton organization. The 
top 10 significantly enriched terms in the BP, CC, and MF categories are shown in Fig. 4a. The KEGG pathway analysis showed that known DE miRNAs target genes were enriched in 124 pathways including pathways for mRNA surveillance and maturity-onset diabetes of the young, and that 14 pathways $(11.3 \%)$ were significantly enriched (Additional file 5). In addition, a scatter analysis was carried out for the first 20 pathways to intuitively show the significance of these pathways (Fig. 4b).

\section{Enrichment analysis of novel DE miRNAs target genes}

A total 1293 target genes from novel DE miRNAs were predicted using the miRanda and RNAhybrid software. GO analysis of novel DE miRNAs target genes showed enrichment of 3138 biological processes (BP), 416 cellular components (CC), and 550 molecular functions (MF), of which 817 BP (26.0\%), 80 CC (19.2\%), and 187 MF (34.0\%) were significant enriched (Additional file 6). Fig. 5a show the top 10 significantly enriched GO terms in the BP, CC, and MF categories. KEGG pathway analysis found 275 enriched pathways including RNA degradation, DNA replication, and pyruvate metabolism (Additional file 7). The top 20 significantly enriched pathways are presented in Fig. $5 b$.

\section{Validation of DE miRNAs}

To validate the sequencing results, we randomly selected six DE miRNAs and examined their expression profiles by q-PCR. The q-PCR results showed that miR-221-3p, miR-9-5p, and miR-142-3p were upregulated, but miR-204, miR-30e, and miR-122-3p were down-regulated (Fig. 6a). These data demonstrated that the trend of miRNAs changes was consistent with the sequencing and q-PCR results.

\section{Discussion}

Rabbits are emerging as a reliable low-cost animal model for human obesity at a time when obesity and associated problems are leading to increased morbidity and mortality [12]. In our experiment, we successfully constructed a rabbit obesity model and selected perirenal adipose tissue for small RNA sequencing. Six randomly selected DE miRNAs for q-PCR validation showed similar trends with small RNA sequencing, indicating our method was reliable and accurately reflected the differential expression of miRNAs between SND and HFD rabbits.

Three SND and three HFD rabbits CDNA libraries were constructed for RNA-seq analysis of DE miRNAs. After processing and analyzing the raw reads, we obtained over $95 \%$ of clean reads, over $99.54 \%$ of Q20, and GC contents greater than $45.74 \%$ indicating that the cDNA libraries were of high quality and the sequenced samples were highly reliable. The distribution of small RNA length of clean reads ranged from $18 \mathrm{nt}$ to $30 \mathrm{nt}$, and the percentage of $22 \mathrm{nt}$ reads was greater than any of the other reads. These results were consistent with research using backfat from male pigs and fat tail sheep [13, 14]. However, other studies found that miRNAs length was influenced by age and tissue. The majority of small RNA-seq reads in the testis of 6 and 12 months old Xiang pigs (28nt to $31 \mathrm{nt}$ ) was much greater than that of 2 and 3 months old Xiang pigs (21 to 23nt), suggesting that the length of miRNAs was different in the testis of immature and mature animals [15]. The dominant size of the small RNA in the testes of breeder cocks 
was $26 \mathrm{nt}$, which was $4 \mathrm{nt}$ longer than the length of miRNAs found in other chicken tissues [16]. These differing outcomes indicate that the length of miRNAs depends on species, tissue, and development period.

We discovered a total of 83 DE miRNAs (52 known DE miRNAs and 31 novel DE miRNAs). There was over a 2-fold differential expression between miR-26a, miR-135a, miR-450b-5p, let-7f-1-3p, novel_mir190, novel_mir154, novel_mir546, novel_mir89, novel_mir208, novel_mir90, novel_mir450, and novel_mir425 from SND and HFD rabbits ( $P<0.001$; Additional file 2 and 3$)$. Some miRNAs were found to be involved in modulation of adipose tissue. For example, the miR-26 family positively regulate adipogenesis and is involved in the regulation of the function of thermogenic adipocytes [17]. Over-expression of let-7i-5p in murine scWAT limited the recruitment of brite adipocytes [18]. Interestingly, miR-9-5p has been reported to regulate white preadipocyte differentiation in culture by targeting the leptin gene[19]. Our findings provided the first evidence for the up-regulated expression of miR-9-5p in the perirenal adipose tissue of HFD-induced obese rabbits. However, the involvement of many miRNAs in regulating fat metabolism needs further in vivo and in vitro experiments is warranted to describe these miRNAs as crucial role in the development of obesity.

miRNAs are able to pair with the 3' untranslated region (UTR) of target gene mRNAs and regulate the expression of the target gene at the post-transcriptional level [20]. A total of 190 known DE miRNAs target genes were predicted using miRanda and RNAhybrid software, and were further analyzed with GO and KEGG to explore their function. The top 10 significantly enriched terms in the BP, CC, and MF categories indicated the possible roles of the known DE miRNAs in regulating adipogenesis. Cytoskeletal remodeling and cell-cell interaction are a necessary step in the transformation of preadipocytes into mature adipocytes, and adipocyte development is dependent on a-tubulin acetylation [21, 22]. Our data showed that regulation of cytoskeleton organization, intermediate filament cytoskeleton organization, intermediate filament-based process, alpha-tubulin binding, cell-substrate adherens junction, anchoring junction, cell-substrate junction, adherens junction, cell junction, intermediate filament, and focal adhesion GO terms were significantly enriched. Furthermore, other GO items related to adipogenesis were also significantly enriched, such as transforming growth factor beta receptor, pathway-specific cytoplasmic mediator activity, and lactate transmembrane transporter activity terms. Increased membrane lactic acid permeability is very important to adipocyte metabolism and is related to adipocyte thermogenesis, browning, and differentiation [23]. KEGG pathway analysis revealed that 14 pathways were significantly enriched (Additional file 5), including the p53 signaling pathway, linoleic acid metabolism, and Wnt signaling pathway. The p53 gene is a tumor suppressor regulator that controls various cellular gene networks. An imbalance between energy intake and expenditure results in excess triacylglyceride accumulation in adipose tissue leading to an increase in oxidative stress and higher expression of tumor suppressor gene p53 $[24,25]$. The linoleic acid metabolism and Wnt signaling pathways are also associated with adipogenesis $[26,27]$. Thus, the known DE miRNAs enriched in these pathways indicate that these DE miRNAs might be an important regulator in adipogenesis. 
GO analysis of the novel DE miRNAs target genes showed that histone ubiquitination, positive regulation of stem cell population maintenance, histone H3-K36 demethylation, cytoplasmic mRNA processing body, poly(A)-specific ribonuclease activity, oxidoreductase activity, acting on $\mathrm{CH}$ or $\mathrm{CH} 2$ groups, oxygen as acceptor, xanthine oxidase activity, oxidoreductase activity, acting on $\mathrm{CH}$ or $\mathrm{CH} 2$ groups, and NAD or NADP as acceptor were significantly enriched. KEGG analysis revealed that the target genes of the novel DE miRNAs were mainly involved in pyruvate metabolism, carbon metabolism, glycerolipid metabolism, glycerophospholipid metabolism, glyoxylate, and dicarboxylate metabolism, suggested DE novel miRNAs might regulate rabbit adipogenesis via citrate cycle (TCA cycle). Future research needs to extensively validate the functions of these novel DE miRNAs and provide a better understanding of their specific mechanisms to regulate adipogenesis.

\section{Conclusions}

we sequenced and analyzed the perirenal adipose tissue miRNAs from rabbits under a standard normal diet and a high-fat diet, and 52 known DE miRNAs and 31 novel DE miRNAs were identified. These DE miRNAs were involved in p53 signaling, linoleic acid metabolism, and other adipogenesis-related pathways. Our results indicate these DE miRNAs might be an important regulator in adipogenesis and contribute to the understanding the epigenetic mechanisms influencing fat metabolism in obese rabbits.

\section{Abbreviations}

SND

Standard normal diet; HFD; High-fat diet; DE:Differentially expressed; GO:Gene Ontology; KEGG:Kyoto Encyclopedia of Genes and Genomes; BMI:Body mass index; WHO:World Health Organization; WAT:White adipose tissue; TG:Triglycerides; BP:Biological processes; CC:Cellular components; MF:Molecular functions

\section{Declarations}

\section{Ethics approval and consent to participate}

All experimental protocols were performed under the direction of the Institutional Animal Care and Use Committee from the College of Animal Science and Technology, Sichuan Agricultural University, China (DKY-B2019202015). The rabbits were humanely sacrificed as necessary to relieve the pain.

\section{Consent for publication}

Not applicable.

\section{Availability of data and materials}


All data generated or analyzed during this study are included in this published article.

\section{Competing interests}

No conflict of interest exists in this manuscript.

\section{Funding}

This study was funded by the China Agricultural Research System (CARS-44-A-2).

\section{Authors' contributions}

Xianbo Jia and Songjia Lai conceived and designed the study; Yanhong Li collected data and conducted the research; Jie Wang and Jiahao Shao wrote the initial manuscript; Mauricio A. Elzo revised the manuscript.

\section{Acknowledgements}

We are very grateful to Tao Tang, Tianfu Lai, Yuan Ma, Mingchuan Gan, and Li Wang for technical support during the experiment.

\section{References}

1. Wang GZ, Du K, Hu SQ, Chen SY, Jia XB, Cai MC, Shi Y, Wang J, Lai SJ. Genome-wide identification and characterization of long non-coding RNAs during postnatal development of rabbit adipose tissue. Lipids in Health and Disease. 2018;17:271.

2. Jones-Johnson G, Johnson WR, Frishman N. Race and Gender Differences in Obesity and Disease. Sociology Mind. 2016;4:233-241.

3. M HC, D FC, D CM, S FD, L OC. Trends in Obesity and Severe Obesity Prevalence in US Youth and Adults by Sex and Age, 2007-2008 to 2015-2016. Jama the Journal of the American Medical Association. 2018;319:1723.

4. Richard, Denis. Cognitive and autonomic determinants of energy homeostasis in obesity. Nature Reviews Endocrinology. 2015;11:489-501.

5. Trayhurn P, Beattie JH. Physiological role of adipose tissue: White adipose tissue as an endocrine and secretory organ. Proceedings of The Nutrition Society. 2001;60:329-339.

6. Li J, Yu X, Pan W, Unger RH. Gene expression profile of rat adipose tissue at the onset of high-fat-diet obesity. American Journal of Physiology-Endocrinology and Metabolism. 2002;282:E1334-E1341.

7. Matsuzawa Y, Nakamura T, Shimomura I, Kotani K. Visceral Fat Accumulation and Cardiovascular Disease. Obesity. 1995;3 Suppl 5:645S-647S. 
8. Haslam DW, James WP. Obesity. Lancet. 2005;366:1197-1209.

9. Du J, Yan X, Zhang P, Xue Z, Gan M, Qiang L, Ma J, Tang G, Jiang Y, Wang J. MicroRNA-125a-5p Affects Adipocytes Proliferation, Differentiation and Fatty Acid Composition of Porcine Intramuscular Fat. International Journal of Molecular Sciences. 2018;19:1-3.

10. He H, Cai M, Zhu J, Xiao W, Liu B, Shi Y, Yang X, Liang X, Zheng T, Hu S. miR-148a-3p promotes rabbit preadipocyte differentiation by targeting PTEN. In Vitro Cellular \& Developmental Biology - Animal. 2018;54:241-249.

11. Zhang XJ, Chinkes DL, Asle A, Herndon DN, Wolfe RR. Lipid Metabolism in Diet-Induced Obese Rabbits Is Similar to That of Obese Humans. Journal of Nutrition. 2008;138:515-518.

12. Dhungel S, Sinha R, Sinha M, Paudel BH, Mandal MB. High fat diet induces obesity in British Angora Rabbit: A model for experimental obesity. Indian Journal of Physiology \& Pharmacology. 2009;53:5560.

13. Pan Y, Jing J, Zhao J, Jia X, Liu W. MicroRNA expression patterns in tail fat of different breeds of sheep. Livestock Science. 2017;207:7-14.

14. Bai Y, Huang JM, Liu G, Zhang JB, Wang JY, Liu CK, Fang MY. A comprehensive microRNA expression profile of the backfat tissue from castrated and intact full-sib pair male pigs. BMC Genomics. 2014;15:47.

15. Luo ZY, Dai XL, Ran XQ, Cen YX, Niu X, Huang SH, Wang JF. Identification and profile of microRNAs in Xiang pig testes in four different ages detected by Solexa sequencing. Theriogenology. 2017;117:61-71.

16. Wu S, Guo W, Li Y, Ren X, Lei X, Li X, Yao J, Yang X. miRNA and piRNA expression profiles of breeder cock testes detected by next-generation sequencing. Reproduction in Domestic Animals. 2017;52:203-213.

17. Karbiener M, Scheideler M. MicroRNA Functions in Brite/Brown Fat - Novel Perspectives towards Anti-Obesity Strategies. Computational and Structural Biotechnology Journal. 2014;11:101-105.

18. Giroud M, Karbiener M, Pisani DF, Ghandour RA, Beranger GE, Niemi T, Taittonen M, Nuutila P, Virtanen KA, Langin D. Let-7i-5p represses brite adipocyte function in mice and humans. Scientific Reports. 2016; 6:28613.

19. Luo G, Hu S, Lai T, Wang J, Lai S. MiR-9-5p promotes rabbit preadipocyte differentiation by suppressing leptin gene expression. Lipids in Health and Disease. 2020, 19:126.

20. Bartel DP. MicroRNAs: Target Recognition and Regulatory Functions. Cell. 2009; 136:215-233.

21. Yang W, Guo X, Thein S, Xu F, Sugii S, Baas PW, Radda GK, Han W. Regulation of adipogenesis by cytoskeleton remodelling is facilitated by acetyltransferase MEC-17-dependent acetylation of atubulin. Biochemical Journal. 2013;449:605-612.

22. Massimo B, Elisa M, Wheeler MB, Li WJ. Transcription Adaptation during In Vitro Adipogenesis and Osteogenesis of Porcine Mesenchymal Stem Cells: Dynamics of Pathways, Biological Processes, UpStream Regulators, and Gene Networks. Plos One. 2015; 10:e137644. 
23. Petersen C, Nielsen MD, Andersen ES, Basse AL, Isidor MS, Markussen LK, Viuff BM, Lambert IH, Hansen JB, Pedersen SF. MCT1 and MCT4 Expression and Lactate Flux Activity Increase During White and Brown Adipogenesis and Impact Adipocyte Metabolism. Scientific Reports. 2017;7:13101.

24. Vogelstein B, Lane D, Levine A. Surfing the p53 network. Nature. 2000;408:307-310.

25. Minamino T, Orimo M, Shimizu I, Kunieda T, Yokoyama M, Ito T, Nojima A, Nabetani A, Oike Y, Matsubara $\mathrm{H}$, et al. A crucial role for adipose tissue p53 in the regulation of insulin resistance. Nature Medicine. 2009;15:1082-1087.

26. Kennedy A, Martinez K, Schmidt S, Mandrup S, Lapoint K, Mcintosh M. Antiobesity mechanisms of action of conjugated linoleic acid. Journal of Nutritional Biemistry. 2009;21:171-179.

27. Christodoulides C, Lagathu C, Sethi JK, Vidal-Puig A. Adipogenesis and WNT signaling. Trends in Endocrinology Metabolism. 2008;20:16-24.

\section{Figures}
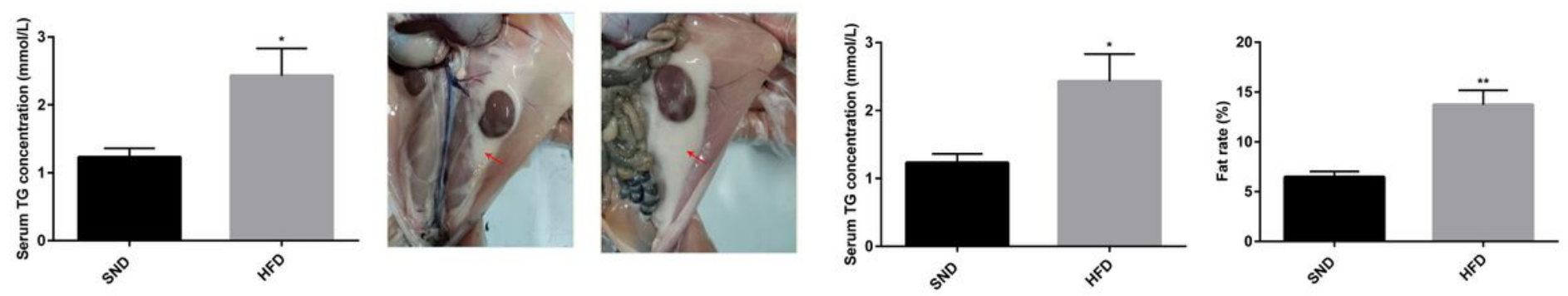

\section{Figure 1}

Establishment of the rabbit obesity model. (a) Body weight of rabbits after 4 weeks on a standard normal diet (SND) and on a high-fat diet (HFD). Images of perirenal adipose tissue in SND (b) and HFD (c) rabbits. (d) Serum triglyceride (TG) concentration in SND and HFD rabbits. (e) Body fat rate in SND and HFD groups of rabbits; $n=3$ for each group, mean $\pm S E M, * P<0.05$, ${ }^{*} P<0.001$. 


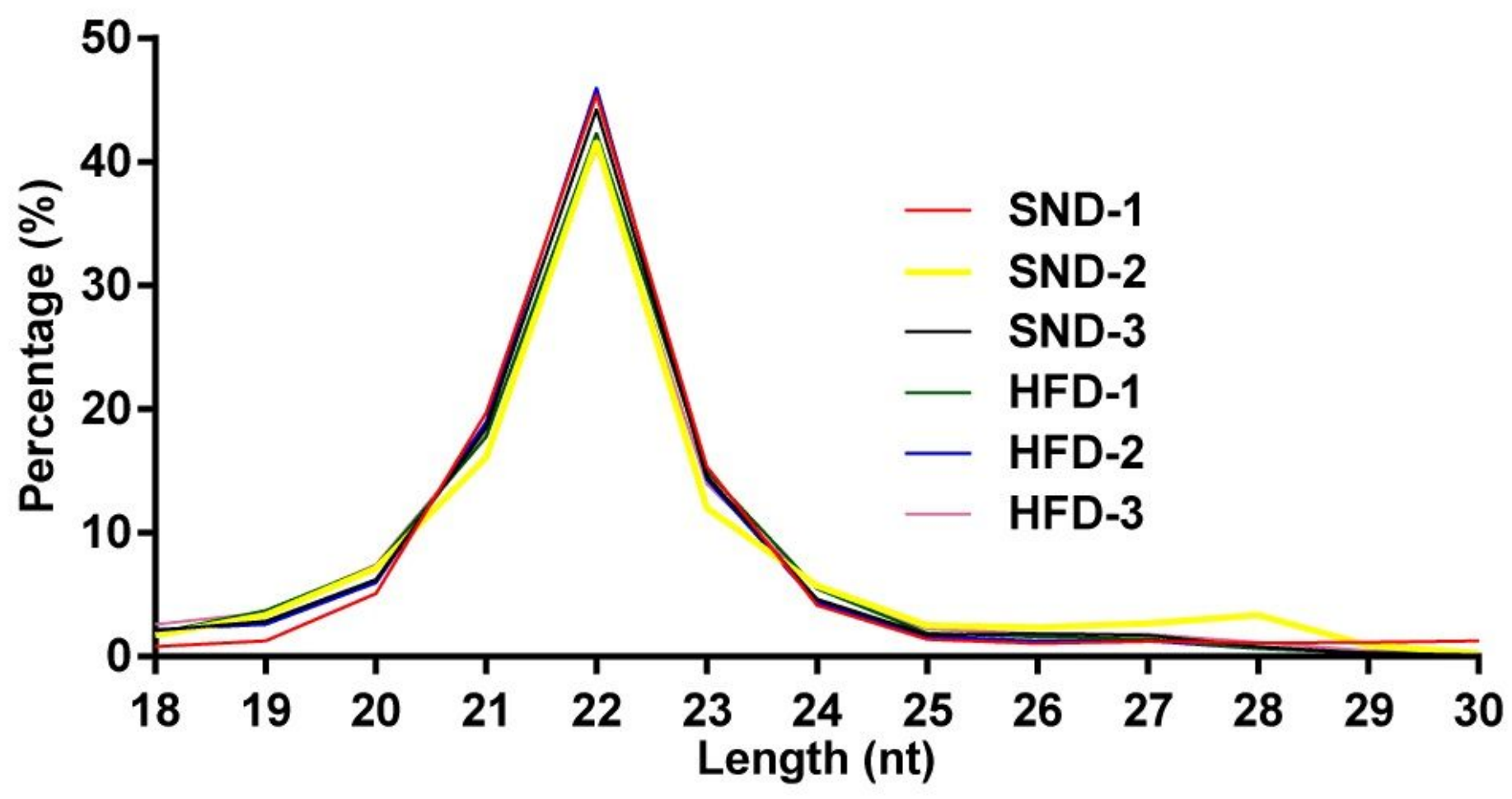

Figure 2

Quality assessment of small RNA sequencing. (a) Distribution of small RNA length for each sample.
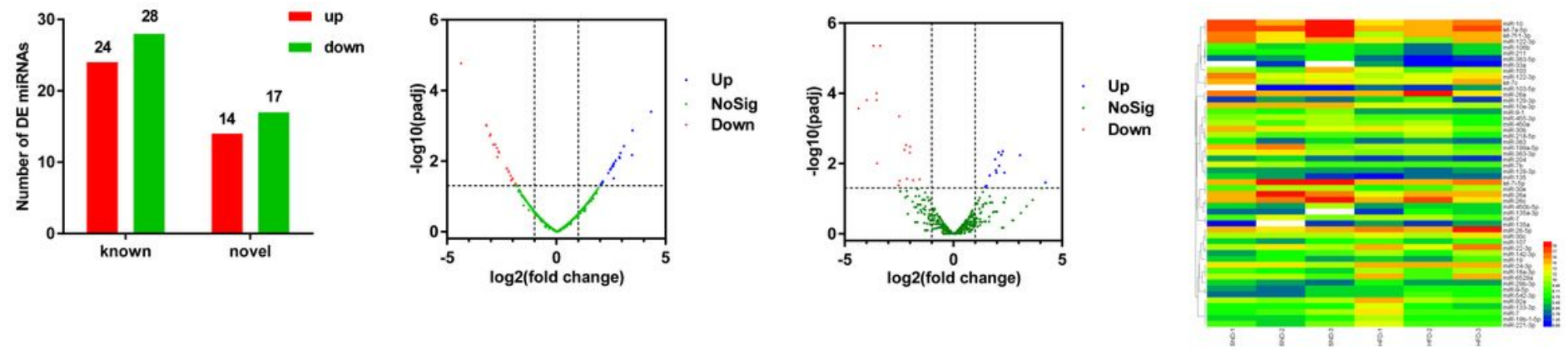

Figure 3

Screening of differentially expressed (DE) miRNAs. (a) Number of known and novel DE miRNAs. (b) Volcano plot analysis of known DE miRNAs. (c) Volcano plot analysis of novel DE miRNAs. (d) Hierarchical cluster analysis of known DE miRNAs in perirenal adipose tissue from standard normal diet (SND) and high-fat diet (HFD) rabbits; red and blue represent high expression and low expression, respectively. 

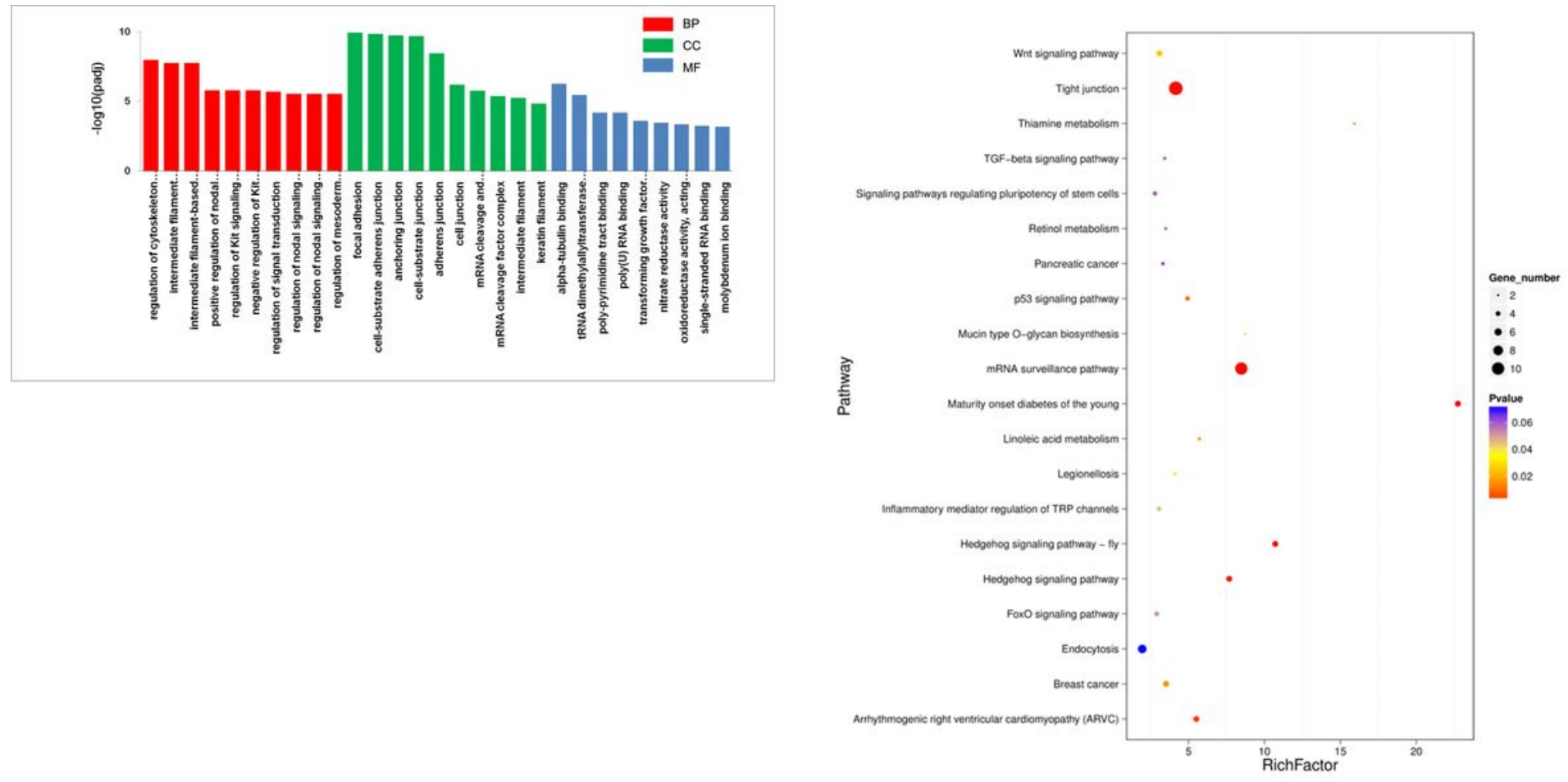

Figure 4

Enrichment analysis of known differentially expressed (DE) miRNAs target genes. (a) GO analysis of known DE miRNAs target genes. (b) KEGG pathway analysis of known DE miRNAs target genes.
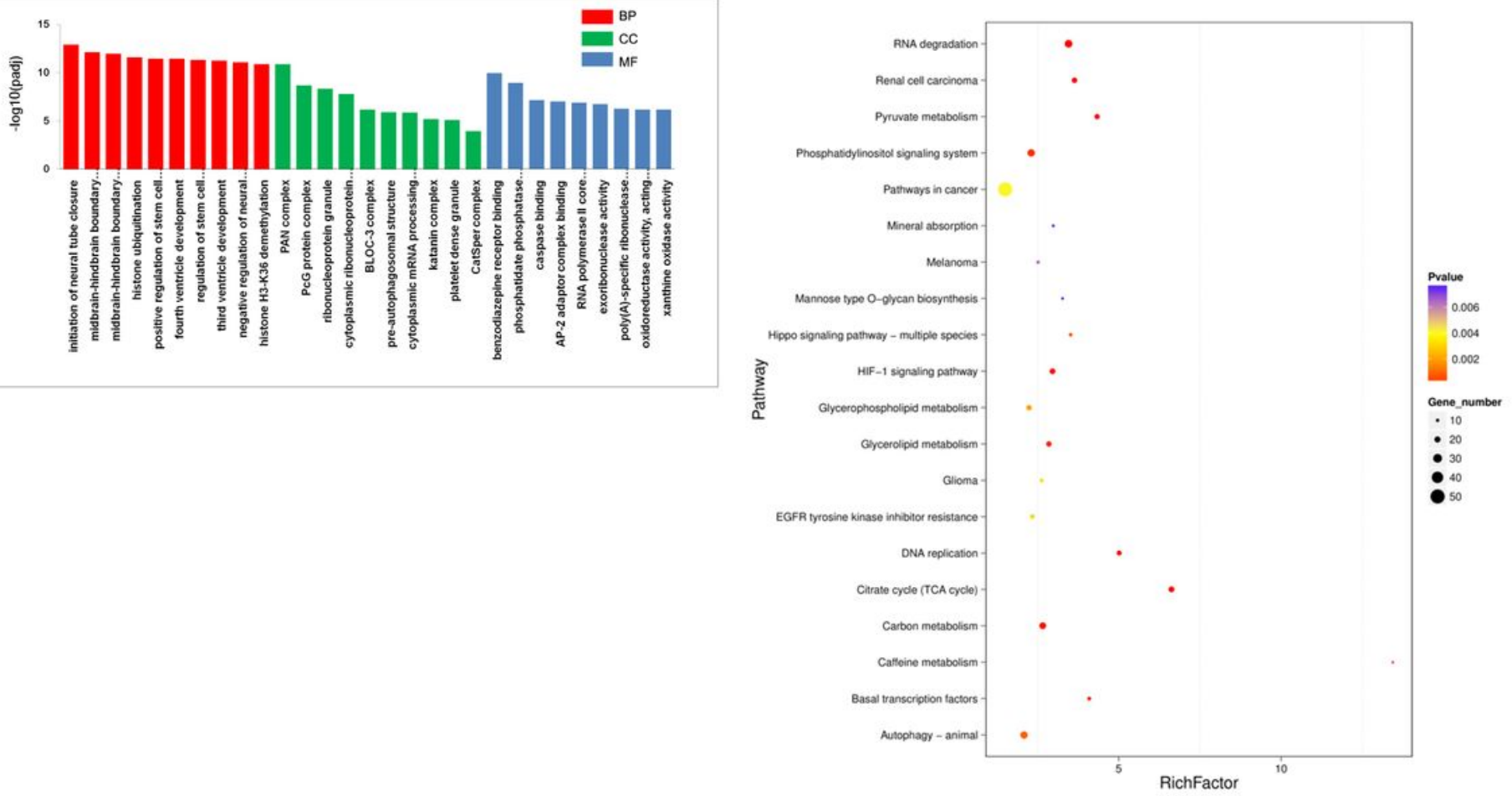

Figure 5 
Enrichment analysis of novel differentially expressed (DE) miRNAs target genes. (a) GO analysis of novel DE miRNAs target genes. (b) KEGG pathway analysis of novel DE miRNAs target genes.

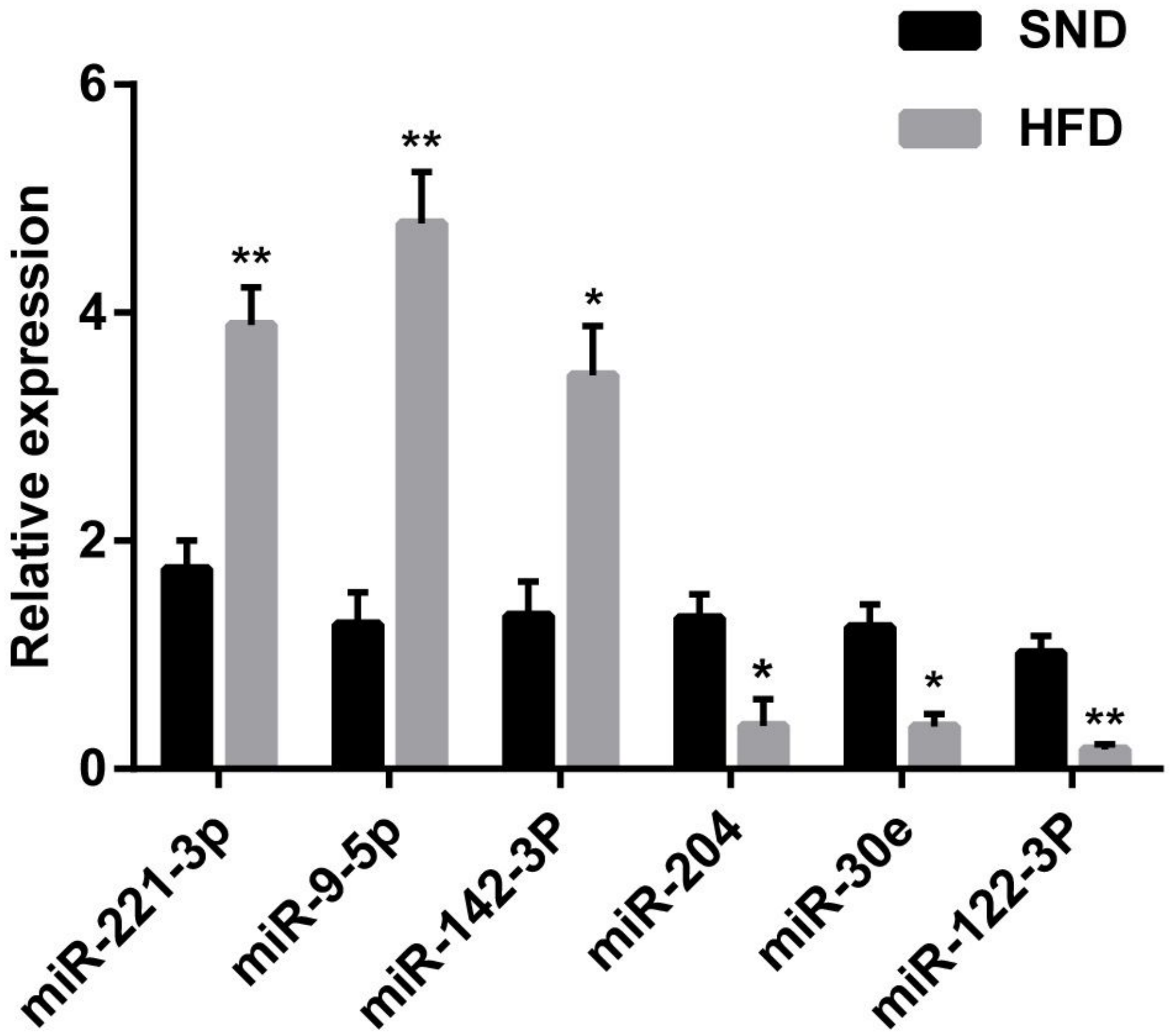

Figure 6

Validation of DE miRNAs. (a) Relatetive expression levels of the six randomly selected DE miRNAs by q$P C R ; n=3$ for each group, mean $\pm S E M, * P<0.05, \star * P<0.001$.

\section{Supplementary Files}

This is a list of supplementary files associated with this preprint. Click to download. 
- Fig5.rar

- Additionalfile6.xIsx

- Fig4.rar

- Additionalfile5.xls

- Additionalfile4.xlsx

- Fig3.rar

- Fig1.rar

- Additionalfile3.xls

- Additionalfile2.xls

- Additionalfile1.xlsx 